\title{
Advancing Measurement Science to Assess Monitoring, Diagnostics, and Prognostics for Manufacturing Robotics
}

\author{
Guixiu Qiao and Brian A. Weiss \\ National Institute of Standards and Technology, Gaithersburg, MD, 20899, USA \\ guixiu.qiao@nist.gov \\ brian.weiss@nist.gov
}

\begin{abstract}
Unexpected equipment downtime is a 'pain point' for manufacturers, especially in that this event usually translates to financial losses. To minimize this pain point, manufacturers are developing new health monitoring, diagnostic, prognostic, and maintenance (collectively known as prognostics and health management (PHM)) techniques to advance the state-of-the-art in their maintenance strategies. The manufacturing community has a wide-range of needs with respect to the advancement and integration of PHM technologies to enhance manufacturing robotic system capabilities. Numerous researchers, including personnel from the National Institute of Standards and Technology (NIST), have identified a broad landscape of barriers and challenges to advancing PHM technologies. One such challenge is the verification and validation of PHM technology through the development of performance metrics, test methods, reference datasets, and supporting tools. Besides documenting and presenting the research landscape, NIST personnel are actively researching PHM for robotics to promote the development of innovative sensing technology and prognostic decision algorithms and to produce a positional accuracy test method that emphasizes the identification of static and dynamic positional accuracy. The test method development will provide manufacturers with a methodology that will allow them to quickly assess the positional health of their robot systems along with supporting the verification and validation of PHM techniques for the robot system.
\end{abstract}

\section{INTRODUCTION}

Since 2000, robots have evolved to enable greater capabilities within a wide range of applications. Robots have been implemented in manufacturing production lines, underwater exploration, hazardous material disposal, space exploration, household usage, entertainment, etc. (Arikan \& Balkan,

Guixiu Qiao et al. This is an open-access article distributed under the terms of the Creative Commons Attribution 3.0 United States License, which permits unrestricted use, distribution, and reproduction in any medium, provided the original author and source are credited.
2000) (H. Chen, Fuhlbrigge, \& Li, 2008) (Park \& Park, 2008) (Summers, 2005). Among the variety of robots, industrial robotics continue to be a significant investment within manufacturing to improve productivity and reduce costs. Industrial robotics are seeing significant growth in recent years. Robot orders and shipments set new records in 2014, increasing $28 \%$ in units and $19 \%$ in dollars over 2013. The automotive industry was the primary driver of growth in 2014, with robot orders increasing $45 \%$ over 2013 (IFR, 2015). Robot systems are providing manufacturers with greater opportunities to improve their productivity, efficiency, and quality. New technologies, such as enhanced sensors and microprocessors, have become more affordable and capable with current robot systems. These changes in robot systems have impacted the applications for which robots can be used within production. For example, arc welding has become a more viable option for various applications, instead of spot welding, due to the improvements in robot system position and trajectory accuracy (S. B. Chen \& Lv, 2014) (Ogbemhe \& Mpofu, 2015). Likewise, as robot systems become more flexible (e.g., capable of 'quick-changing' different end-effectors to perform a range of tasks within the same work cell), they are becoming more capable of meeting customer demands for greater product variety in small volumes (Muller, Esser, \& Vette, 2013) (Bi \& Lang, 2007).

As robotic technologies become more integrated with complex manufacturing environments, robot system reliability has become more critical. Health monitoring, diagnostics, prognostics, and maintenance (collectively known as Prognostics and Health Management (PHM)) have gained more attention within the robot system domain with respect to the design, implementation, operations, and maintenance phases. Advanced robot system manufacturing processes are raising the demands on PHM capabilities, thereby requiring an evolution of PHM performance assessment techniques. Reconfigurable manufacturing systems are becoming more mainstream to handle high variety/ low volume production demands (ElMaraghy, 2005). More frequent reconfigurations of the robot system force the 
available PHM techniques to be implemented more frequently, be more granular, and be more accurate. PHM for robotics research needs to keep pace with the latest innovations to support emerging robot systems within these flexible operational scenarios.

PHM references the cluster of strategies and techniques that promote condition monitoring, diagnostics, prognostics, and maintenance of a product, machine, or process. PHM technologies are applied to manufacturing systems to reduce unscheduled downtime and costs. PHM can be applied to both products and processes.

A product is defined as a system, usually composed of hardware and software, that is designed to carry out a specific function or set of functions. Product PHM is typically focused on a physical entity (Malinowski et al., 2015). An example of product PHM would be monitoring the health of the robot arm itself. Product PHM (e.g., within the automotive, aerospace, and power generation communities) is more available as compared to process PHM (e.g., found in machining, welding, packaging, machine-tending, and assembly manufacturing operations (Batzel \& Swanson, 2009) (Denkena, Litwinski, Brouwer, \& Boujnah, 2013) (Holland, Barajas, Salman, \& Zhang, 2010) (Hu \& Koren, 1997) (Lee et al., 2014) (Malinowski et al., 2015) (Shen, Wan, Cui, \& Song, 2010) (Siegel, Lee, \& Dempsey, 2014) (Siegel, Zhao, Lapira, AbuAli, \& Lee, 2014).

A manufacturing process is typically composed of one or more pieces of equipment, technology, and human resources acting together with energy and material to perform an overall task that yields an output. An example of process PHM would be monitoring the health of an overall robot system that is tasked with pick and place operations.

The big distinction between a product and a process is that a product's sub-systems and components typically interact in the same manner throughout its lifecycle to perform the same function whereas a processes sub-systems and components may be replaced and/or reconfigured to dramatically alter the nature of the process. Ultimately, a product's configuration is likely to remain static over time whereas a process's configuration is likely to evolve as new sub-systems and components (e.g., emerging robotic arms, additive manufacturing equipment replacing subtracting machine tools) are introduced into the process. This means that any integrated PHM must evolve, accordingly. Many of the existing PHM strategies are adept at handling product PHM; fewer PHM techniques are capable of being integrating into the sometimes volatile nature of the manufacturing process. PHM can become a necessity in the face of faults and failures depending upon the frequency, severity, and recovery time from the fault or failure. Not all faults and failures are equal, especially when it comes to manufacturing robotics.

\section{FAULTS/FAILURES OF INDUSTRIAL ROBOT SySTEMS}

\subsection{Classification of Faults and Failures in Industrial Robot Systems}

Industrial robot systems are complex. They are composed of many components including arms, end-effectors, fixtures, sensors, and safety systems. There are many possible faults and failures that could occur within the robot system given this system complexity. It is critical to determine the types of faults and failures manufacturing PHM robotics research should focus to ensure alignment with industry needs and priorities (National Institute of Standards and Technology, 2015).

Faults and failures can be divided into three principal categories (Caccavale, Marino, Pierri, \& Ieee, 2010) (Bittencourt, 2012) (Wang, Liu, \& Xu, 2008): faults, soft failures, and hard failures.

A fault is defined as a defect, an incorrect signal value, or incorrect decision within the system. It is an inherent weakness of the design or implementation. A fault may cause a system's degradation to be accelerated resulting in a failure (Parhami, 1997). When the system is in a fault condition, minor damage can occur that can lead to the accumulation of wear and tear to the system. Productivity and output product quality may still be achieved. The system's health status may still be considered acceptable.

A soft failure indicates the occurrence of degradation, 'wear and tear,' and/or external changes that have damaged something within the system. A soft failure is a condition under which the process can still be performed, but the system performance starts to degrade. Under a soft failure, the process is not capable of meeting its performance objectives (e.g., instead of a process manufacturing 50 cars/hour, it is only able to make 40 cars/hour and/or the quality of the cars is degrading) (Yamada \& Takata, 2002). As the performance degradation gets worse, quality can ultimately decrease to the point that it is below the necessary specifications (e.g., cars are being made, yet they would be considered scrap since their quality is below the necessary specification).

A hard failure is indicative of broken component/piece of equipment, or the inability of a system or component to perform its required functions within specified performance requirements (Parhami, 1997). In a hard failure condition, the manufacturing process is compromised where it is no longer capable of producing the necessary products to specification. The manufacturing process is typically either frozen or shut down.

The aim of PHM technologies and strategies is to avoid both soft and hard failures. Depending upon the nature of a failure and the available monitoring, failures may first be noticed at the system level or the component level. Given the vast research that has focused on component monitoring and failures, system-level monitoring and failures are an identified research challenge. System-level intelligence will 
be used to trace back the root cause to the origination of the failure at whatever level it may have occurred.

Soft failures usually come from environmental parameter changes (including thermal conditions, control code modifications, or preload changes), improper installation (which can cause performance degradation), atypical disturbances during normal system operations, and through normal wear and tear (Massi et al., 2014) (Agheli, Qu, \& Nestinger, 2014) (Bittencourt, 2012). System-level soft failures can originate from a component-level hard failure, in turn which can be caused by normal wear and tear. For example, preload changes may influence the positional accuracy of the robot system's tool center point. Temperature changes can influence the accuracy of external sensors (e.g., vision sensors that are used to aid the positioning of parts relative to a robot arm).

\subsection{Impacts of Faults and Failures}

Faults and failures can impact a manufacturing process in numerous ways including impacting some key performance metrics of a robot system including:

Accuracy - Defined as the measurement of the deviation between the commanded and attained robot position and orientation (Shirinzadeh, 2000). Accuracy can also represent the difference between commanded and actual velocities, accelerations, forces, and torques.

Likewise, faults and failures can impact some common robot specifications including:

Velocity - Defined as the vector (rate and direction) at which the robot tool center positon (TCP) changes its position (Abdi, Nahavandi, Frayman, \& Maciejewski, 2012)

Force - Defined as the push or pull upon an object resulting from the robot TCP's interaction with the object (Niku, 2011)

Torque - Defined as the rotational moment on an object. measured at the individual joints or at the TCP, if possible (Niku, 2011)

These metrics are commonly identified as critical indicators of system performance and health. For example, accuracy is a critical performance factor for applications that demand both positional and trajectory accuracy (e.g., arc welding). Accuracy is also critical for applications that are using an external system to position parts relative to the robot arm, or using an external system to guide a robot's operation (e.g., a vision system may be used to locate a part; this information would be passed to the robot controller that would then command the robot to pick it up) (Zhang, Yan, Zhu, \& Wen, 2012). If accuracy is degrading, a robot might drill or weld at incorrect positions. Product quality could become compromised (but because the robot system is still operating without being frozen or broken, the soft failure may be difficult to immediately detect). The degradation of the robot system's accuracy will decrease the quality of manufacturing and reduce production efficiency. Given the high output rate of production lines, it is critical to develop techniques to verify and validate robot system health assessment techniques that predict soft and hard failures. Another example is that in the condition of torque degradation, the controller cannot get precise torque sources and may continue to drive the actuator until it is damaged (Liu, 2001) (Tsui, Chen, Zhou, Hai, \& Wang, 2015). Performance degradations are less visible compared to system freezes or shutdowns because the system is still performing its tasks, but at a decreased level of performance and/or quality.

Hard failures usually come from mechanical failures (e.g., damage of mechanical parts, actuators and/or sensor failures), power system failures, or controller unit failures. Hard failures will stop the current process until the failures are resolved (Kahan, Bukchin, Menassa, \& Ben-Gal, 2009). Hard failures are more visible because production is interrupted/stopped. Resolving hard failures requires some form of maintenance.

\section{NEEDS OF MANUfaCtURERS}

Enhancing accuracy within manufacturing robotic operations would be greatly beneficial to the manufacturing community in terms of improving their efficiency and product quality while reducing scrap. Developing, advancing, and integrating monitoring, diagnostic, and prognostic capabilities will support these enhancements. However, there are numerous technological challenges that must be addressed to increase the capability of PHM, ultimately leading to improved accuracy.

\subsection{Improving Accuracy}

There is a growing demand within the automotive and aerospace industry for greater robot accuracy (Buschhaus, Blank, Ziegler, \& Franke, 2014) (Young \& Pickin, 2000). For example, there are three options for welding within the automotive industry: spot welding, arc welding, and laser welding (Kusuda, 1999). Spot welding used to be the most widely used welding method even though the other two options offer significant benefits (e.g., arc welding and laser welding are continuous welding processes which create higher welding speeds, thereby increasing productivity). The reason spot welding is predominantly used is because it does not require the finer accuracies that are necessary for the other two methods. An accuracy of $5 \mathrm{~mm}$ is needed for spot welding. Arc welding requires an accuracy of $0.5 \mathrm{~mm}$. Laser welding requires an accuracy of $0.1 \mathrm{~mm}$ (Wilson, 1999). Since arc and laser welding conduct their operations through continuous paths, the system's capabilities to control repeatability, accuracy, velocity, force, and/or torque are key metrics for the robot system's performance.

Recent accuracy improvements have greatly enhanced aerospace manufacturing capabilities. High robot accuracy during manufacturing ensures that parts are precisely manufactured with predictable results even after changes are 
made to the process. Systems that combine processes like drilling, assembly, routing, and material removal require both positional accuracy and path accuracy. High accuracy robots are becoming valuable tools for many of the afore-mentioned processes. Enhanced robot accuracy can lead to substantial cost savings for the aerospace industry (Jeffries, 2013) (DeVlieg, 2010).

High accuracy is also critical in data-driven applications, such as those applications developed using off-line programming methods (Pan, Polden, Larkin, Van Duin, \& Norrish, 2012) (Mitsi, Bouzakis, Mansour, Sagris, \& Maliaris, 2004). In aerospace applications, large volumes of precise holes must be drilled into airframes (DeVlieg, 2010). Significant production time can be saved by applying off-line programming, because the actual manufacturing would only have to be interrupted briefly, while the new programs are downloaded into the control computers. Moreover, the market requirement for high design-variation and low-batch production has meant that users are looking more towards off-line programming.

Another application that inherently requires a high degree of accuracy is online inspection or gauging which sometimes calls for robots to be part of the measurement operations (Edinbarough, Balderas, \& Bose, 2005) (Ngan \& Tam, 2004). There are a large number of automotive and aerospace applications that currently or could utilize the flexibility of robotics to perform metrology on manufactured parts. For example, automotive body panels are routinely and painstakingly measured and compared to a known standard. This standard could be a known, "golden" part. A measurement device could be mounted to the end-effector of a robot that moves this device into positon to take a measurement. All measurements are related back to robot position; the measurement accuracy will only be as accurate as the robot positioning. For example, X, Y, Z, pitch, yaw, and roll need to be measured to account for a system's accuracy. There has been a lack of measurement science (defined as performance metrics, use case scenarios, test methods, reference datasets, and software tools to promote unbiased assessment) to verify and validate position and trajectory accuracy health assessment strategies. Measurement science techniques need to be developed to assess the presence of soft failures.

\subsection{PHM Challenges}

The National Institute of Standards and Technology (NIST) hosted the Roadmapping Workshop - Measurement Science for Prognostics and Health Management for Smart Manufacturing Systems in Fall 2014 to examine the needs and priorities of stakeholders to advance key PHM technology areas, including monitoring, diagnostics, and prognostics, with an emphasis on manufacturing operations (Weiss et al., 2015). The results of the workshop presentations, panel discussions, and breakout sessions were captured in a comprehensive workshop report (National Institute of Standards and Technology, 2015). The workshop report identifies numerous priority roadmap topics that present a broad view of the PHM research landscape; many of these topics are relevant to manufacturing robotic systems. While the workshop report presents substantial detail of each roadmap topic, the remainder of this section will present several key topics for examination within the robotics domain.

- Advanced Sensors for PHM in Smart Manufacturing - a complete understanding is lacking with respect to the full suite of sensing capabilities, interfaces, and interoperability requirements necessary to successfully fuse PHM with manufacturing robotic operations. Addressing this topic includes developing an inventory of current sensors and identifying gaps for PHM, within manufacturing robotics (i.e., leveraging PHM to improve accuracy), with regard to configuration, flexibility, and other factors.

- $\quad$ PHM Data Taxonomy and Architecture - there is a lack of interoperability for sensor/data formats and communication modes to capture, share, and analyze data across heterogeneous robotic systems. This challenge can be remedied through the creation of 1) a data taxonomy for PHM that covers data formats, storage, organization, semantics, and other pertinent elements and 2) standard data interfaces and communication protocols. Communication protocol standards are already in use with numerous machine tools and a range of relevant sensors where it would be advantageous to expand this capability to robotic systems(Vijayaraghavan et al, 2008).

- Overarching Architecture Framework for PHM with Standards and Key Performance Indicators (KPIs) with respect to PHM performance assessment, industry is absent standards or guidelines enabling various PHM technologies, as applied to robotic systems, to be evaluated in unbiased manners. This challenge can be solved through the development of a framework that would include benchmarking current states, determining key performance indicators, and defining a standard architecture aimed at performance assessment and traceability.

- Cost Model for PHM Performance - the PHM stakeholder community is currently devoid of methods that can accurately predict the cost savings of a PHM system based upon gathered risk data along with other key characteristics. Overcoming this challenge requires cost data for equipment failures (e.g., failures of endeffectors, sensors, robot arms), collateral damage (e.g., damage done to parts or fixtures), and lost opportunities (realized upon unscheduled downtime).

- Identification of PHM Performance Metrics - the manufacturing robotics community does not currently 
have standard guidance as to the critical performance metrics that adequately present a robot system's health status along with diagnostic and prognostic information about what will fail and when it will happen. Performance metrics need to be identified that present a relatively complete picture of robot system health under varying conditions (e.g., one set of performance metrics may be sufficient when a robot is relatively healthy while another set of performance metrics may be necessary when the robot system is in a lesser health state).

- Failure Data for Prognostics and Diagnostics - the manufacturing robotics community does not have access to relevant, accurate, and appropriately contextualized fault and failure data to support the development of diagnostics and prognostics. Likewise, formats of existing data sets are largely inconsistent making them challenging to integrate and leverage. Addressing this shortcoming requires the development of test beds and data collection methods that would focus on generating, verifying, and validating fault and failure data for public consumption.

The afore-mentioned roadmap topics are a subset of the documented topics from the workshop report and are highlighted by their applicability to manufacturing robotic operations.

To further understand the manufacturing robotics landscape, NIST personnel met numerous small, medium, and large manufacturers to hear specific details of where PHM can be leveraged or improved to enhance asset availability and operations efficiency. These efforts have been documented (Jin et al., 2016) and have been folded into NIST's PHM research discussed in the following section.

\section{AdVANCING PHM ASSESSMENT FOR MANUFACTURING ROBOTICS}

Research is being conducted at NIST to advance the capabilities to verify and validate monitoring, diagnostic, and prognostic technologies to enhance maintenance and control strategies within manufacturing operations. Specifically, the research aims to develop performance metrics, test methods, reference datasets, and supporting tools that the manufacturing community can leverage to enhance their PHM capabilities (Weiss et al., 2015). PHM for robotics is part of this research effort. This research area focuses on the developing verification and validation techniques to assess the capabilities to monitor, diagnose, and predict failures impacting a robot's accuracy with respect to several specifications (e.g., position, velocity, force). The robotics research plan is highlighted in Figure 1. It is important to note that although NIST identified a wide-range of research challenges regarding manufacturing robotics PHM, the NIST research team is only addressing a subset of these topics based upon available resources.
NIST has organized their research into three stages. Stage one focuses on the development of research goals and objectives. Stage two focuses on the development of specific measurement science products. Stage three is highlighted by technology transfer efforts with industry and standard development organizations (SDO).

As shown in Figure 1, information-gathering and gap analyses are performed to identify the key measurement science required for this research within the first stage (Weiss et al., 2015) (Jin et al., 2016). Interactions are underway to collaborate and partner with various external stakeholders including manufacturers, technology developers, and SDOs to better align our research and development (R\&D) efforts and standards activities.

During the second stage, the research is focused on the development of measurement science products. Two categories of performance metrics in PHM measurement science will be identified - 1) the metrics used to assess the health of the system (e.g., accuracy of the TCP) and 2) the metrics used to verify and validate the PHM techniques (e.g., accuracy in terms of predicting when something is going to fail vs. when it actually does fail). Furthermore, test methods (defined as operationally-relevant, relatively-controlled situations to support technology assessments) are being developed to assess the different health metrics of robot systems. It is important to note that only a subset of the overall potential metrics will be the initial focus of this activity. It is expected that accuracy of the TCP will first be examined, followed by accuracy of the tool center's velocity (sometimes referred to as path accuracy). Additional metrics will be added in future efforts.

A PHM for Robotics test bed is being constructed to provide a platform for the development, testing, verification, and validation of the planned PHM for robotics measurement science output. The test bed will serve as the home to several industrial robot arm systems and will promote the generation of operationally-relevant test methods and datasets (Weiss et al., 2015). Advanced sensing and data collection techniques (what information to collect, how to collect, sensors to use, etc.) will be developed. Reference datasets will be generated to offer researchers and manufacturers a means of verifying and validating their diagnostic and prognostic techniques without the need for their own physical implementations. Reference data processing algorithms (data synchronization, data fusion of multiple sensor streams, and PHM data formats for interoperability) will be developed to analyze the PHM data that assesses the robot system's health metrics. The verification and validation methods of the PHM techniques will be designed. A closed-loop solution (a control system in which an operation or process is regulated by feedback) is being developed to support the generation of verification and validation techniques of robot system control by feeding back various solutions (i.e., updated programmable logic 


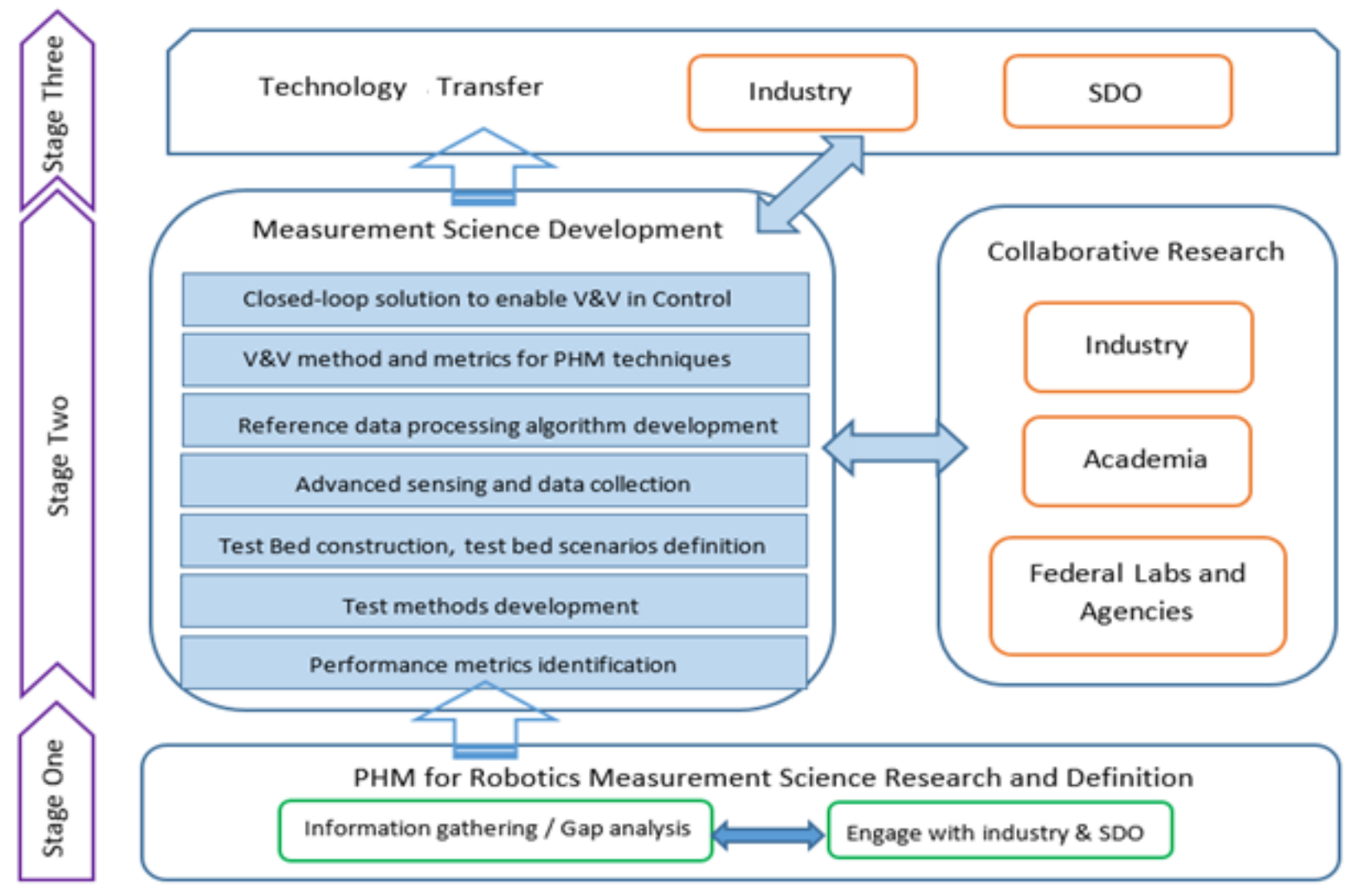

Figure 1. PHM for Robotics research plan

controller (PLC) control strategies, maintenance recommendations). Also in stage two, collaborations are further developed with stakeholders in the industry, academia, and research communities to ensure our latest information on emerging PHM techniques is accurate and, in turn, receive feedback on the initial development of measurement science products.

The third stage of this research will focus on standards development and technology transfer to the manufacturing community. The ultimate goal of this work is to promote the development of appropriate guidelines and/or standards that enable manufacturers, technology developers, and technology integrators to appropriately select, deploy, and assess their PHM for robot systems capabilities within factory operations.

The output test methods, techniques, and supporting tools are intended to be adopted by industry for PHM applications and become the technical underpinnings of published guidelines and/or standards. Through the development of these measurement science products, manufacturers can access effective tools to verify and validate their monitoring, diagnostic, prognostic, and maintenance capabilities. If applied, these outputs are expected to reduce unexpected downtime, improve productivity, efficiency, and quality, and transition maintenance from reactive to preventative to predictive to proactive maintenance.

Use cases will be created within the overall test bed. The first use case is the development of a robot system quick health assessment methodology. This use case develops a test method (which is part of the methodology) that focuses on the positional accuracy, including static accuracy and dynamic accuracy (accuracy of trajectory/velocity). This test method will enable manufacturers to quickly assess the static and dynamic accuracy of their robot systems when environmental conditions change, after the work cell has been reconfigured, or whenever a manufacturer wants to determine if they have experienced a degradation. The use of this methodology will improve productivity and reduce unexpected shut-downs, and help transition maintenance from reactive, to preventative, to proactive maintenance. Additional use cases are actively being developed and will be documented in future publications.

The key building blocks of the PHM for robotics structure are shown in Figure 2. The first key building block is the advanced sensing module for PHM (shown in the upper left of Figure 2). Advanced sensing will be developed to measure and monitor the system's health status. Advanced sensing will have three layers: a system layer, a component layer, and an add-on layer. System layer sensing aims to support the 


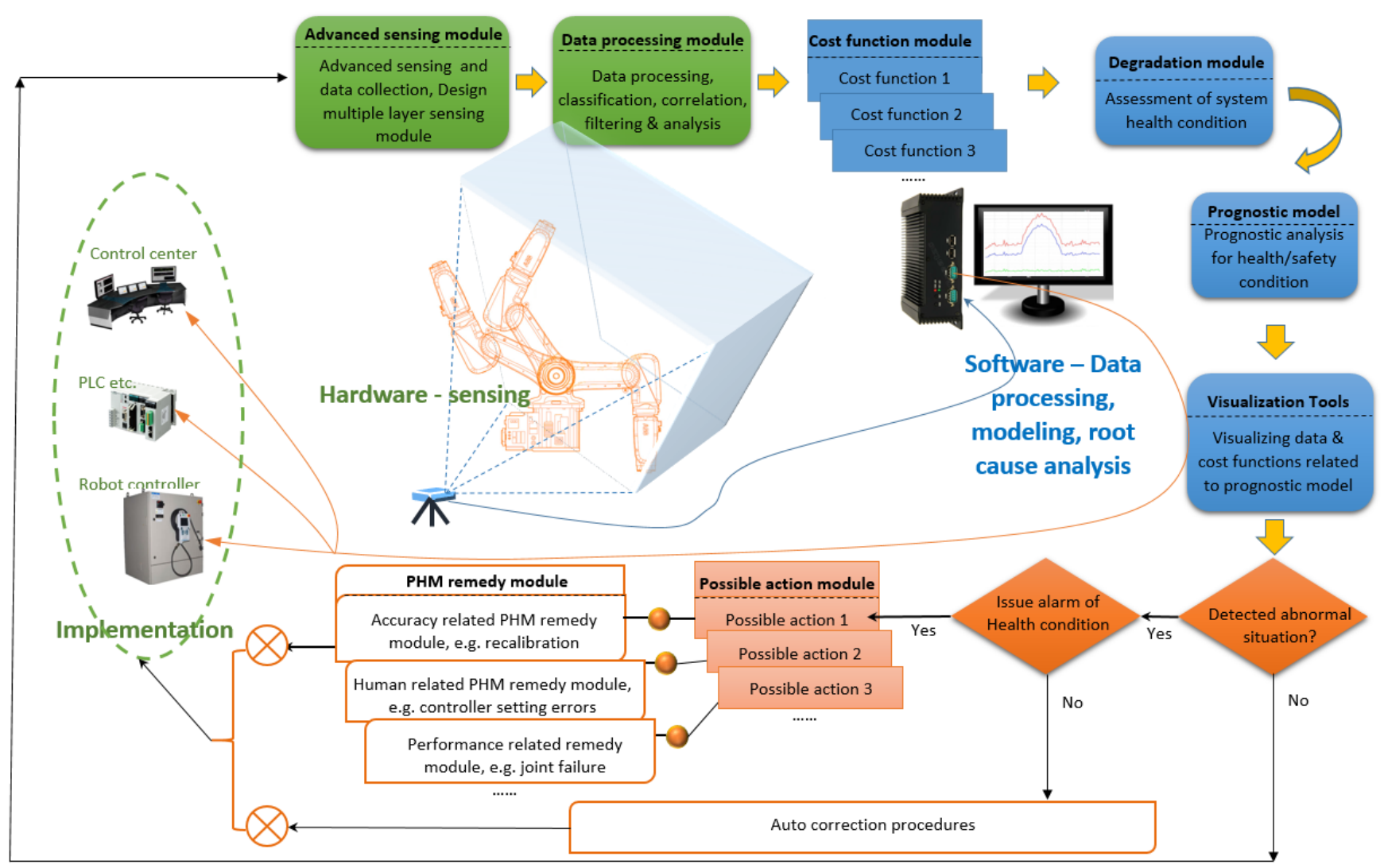

Figure 2. Key building blocks of the PHM for Robotics structure

overall system's health assessment. The first test methods will focus on the system's static and dynamic accuracy (i.e., positional accuracy test methods); the component layer sensing extracts data from the robots' controllers and/or embedded sensors to perform the on-line monitoring; the add-on layer promotes the inclusion of additional sensors to provide information that the component and system layers may be neglecting. The second key building block is the data processing module (shown as the data processing module in Figure 2). This module will center on the development of reference algorithms to fuse data captured from multiple sensors employed in the advanced sensing module. The data processing module will offer greater analysis capability through targeted data collection on top of complex and/or reconfigurable robotic applications. The third key building block is the development of algorithms for robot system health assessment and PHM verification and validation methods (collectively shown in the cost function module, degradation module, prognostic module, and visualization tools module in Figure 2). As the fourth key building block, the closed-loop implementation of PHM solution within the control structure is reviewed.

This structure serves as the back bone of use case development. The development and expansion of each module will further address elements of the measurement science. It also serves as the platform for reference datasets collection.

\section{Development of USE CASE 1 - Methodology ON QUICK HEALTH ASSESSMENT}

The first use case is the development of a quick health assessment methodology to identify the health of the robot system, with an emphasis on the subset of the robot health performance metrics - static accuracy (accuracy of position) and dynamic accuracy (accuracy of trajectory/velocity). This effort will provide manufacturers with a methodology that will allow them to quickly assess the TCP health of their robot systems when environmental conditions change, or after the work cell has been reconfigured. In turn, this methodology can also allow manufacturers and technology developers to verify and validate their own PHM techniques that monitor robot health in terms of static and dynamic accuracy.

The quick health assessment methodology contains the development of test methods, sensors used to take measurements, reference algorithms for data processing and health assessments, and verification and validation $(V \& V)$ of PHM techniques. The test bed environment to develop this methodology features a single six degree of freedom (DOF) robot that is mounted to an optical table. The robot is hard- 
coded to move its TCP in a fixed loop motion that is influenced by the test method (further details are provided in Section 5.1). Given the geometry and kinematics of the expected test bed industrial arm robot, it would take approximately five minutes for this test method to be fully executed (the location of each point is a function of the robot's geometry and kinematics). While the TCP is moving to these pre-determined positions, $\mathrm{X}, \mathrm{Y}, \mathrm{Z}$, pitch, yaw, roll, and time data are being captured from a seven dimensional (7-D) measurement system (described in Section 5.2) This measurement is used as the system layer sensing data for PHM because they directly reflect the final accuracy of the robot system. For this methodology development, the component layer data is also extracted from the robot controller, including actual joint position, target joint position, actual joint speed, actual joint current, joint motor temperature, etc. Moreover, a set of add-on sensors with wireless communication are also designed for add-on layer data to get additional orientation information of each robot arm. The component and add-on layer data are used to promote root cause analysis once faults and failures are detected from the system layer sensing. Further, the V\&V methods are developed for the measurement system and test methods. This is to ensure that the accuracy of the measurement system is qualified for the tasks of measurement. The V\&V of the test method's accuracy are developed in terms of prediction of when and where faults and failures are going to occur. Selected items are further discussed in the following sections.

\subsection{Test Method Development}

A test method is developed to assess the robot system health of positional accuracy, which is a subset of robot system performance metrics. The robot system's positional accuracy includes the robot arm's accuracy and the accuracy of any system interacting with the robot arm (e.g., a conveyor moving products within range of the robot arm). To assess their positional accuracy, all measurements will be taken under a global coordination system which is defined on the 7-D measurement system. In this case, assessment of the positional accuracy of the robot arm or any interacting systems are respectively referred to in this global coordinate system. A detailed description of the robot arm test method development is provided.

To access the robot arm's positional accuracy health status, a fixed loop motion is developed. While the TCP is moving to these pre-determined positions, $\mathrm{X}, \mathrm{Y}, \mathrm{Z}$, pitch, yaw, roll, and time data are being captured from a 7-D measurement system (details in Section 5.2). Analyzed position, time, and orientation data will provide a measure of the positional accuracy of the robot system when compared to original specifications and prior measurements. Ideally, periodic data would be collected to track accuracy degradation with minimal disruptions to production. This accuracy degradation data would offer insight into the robot system's health.

This fixed loop motion of the robot arm is designed such that the test method can be executed in a relatively short amount of time. The test method aims to assess the health of the robot arm's positional accuracy by capturing and analyzing the accuracy of robot arm in Cartesian space. A set of specific points are designed in Cartesian space that covers a range of robot arm poses, including some that are near, far, high, and low (shown in Figure 3). The inclusion of these poses is purposeful so as to exercise the full range of the robot arm and evaluate arm accuracy and rigidity when the arm is both fully and minimally extended. At position 1 and position 11, motions are added that will exercise robot joints at different combination angles while keeping the robot TCP at a fixed position. This type of motion is particularly sensitive to robot axes offset errors. We use this motion to detect offset changes between linkages.
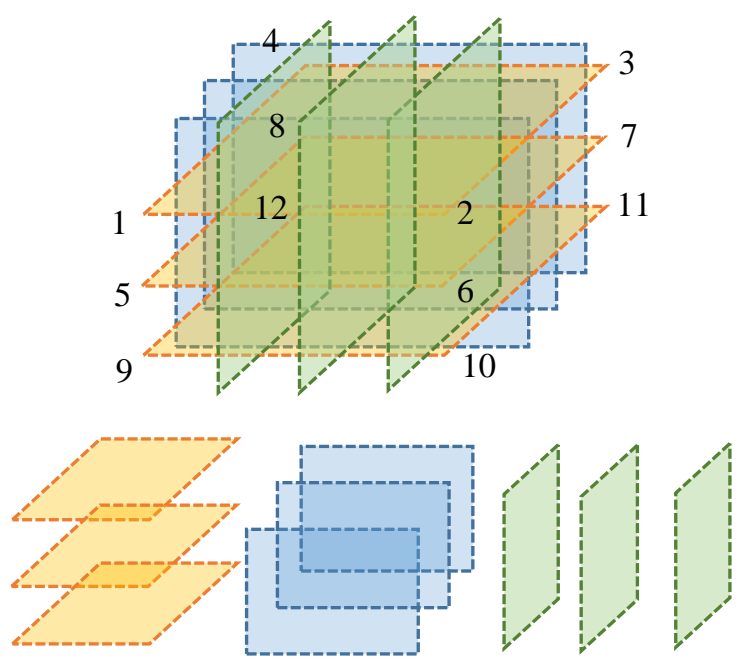

a) Path of the fixed loop motion

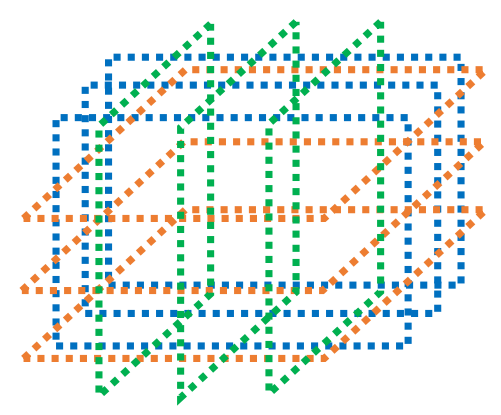

b) Measurement points from the fixed loop

Figure 3. Fixed loop motion of quick health assessment

The fixed loop motion consists of movements along the edges of three horizontal planes, three vertical planes, and three planes perpendicular to the previous two sets of planes. For 
example, when the robot is moving along the first path of horizontal planes, the robot arm moves continuously from position 1 to position 2 , then to position 3 and finally to position 4 . The final measurement result is a dense point grid, as shown in Figure 3b). Each grid position contains the information of time, position, and orientation.

Several features of this test method are highlighted:

- For the robot movement programming, the fixed loop method commands the robot to move in Cartesian space. Robot kinematics are used to calculate accuracy deviations in Cartesian space.

- Instead of 'move-and-pause' static measurement, the fixed loop motion commands the robot arm to move continuously. This promotes continuous data sampling from the measurement system. In this case, dynamic performance of the robot arm is also captured. We can achieve static measurements through the average of continuous data sampling.

- The measurement system needs to be designed such that its integration and use does not interfere with the robot system's normal operations. This includes avoiding the scenario where a robot system's end-effector needs to be removed or adjusted to accommodate a sensor.

- The add-on system should be easy to integrate to avoid complex wiring and integration with the system's controller(s).

- The system should be a relatively low-cost solution for industrial implementation.

Existing six dimensional (6-D) technology (X, Y, Z, pitch, yaw, and roll) measurement systems include laser trackerbased systems and vision-based systems. The laser trackerbased system needs to maintain line-of-sight between the laser tracker and target. The target usually needs to be mounted on the center of TCP (this requires changing setups or work tools). Laser tracker-based systems are relatively expensive. Optical tracking uses the principles of modern high-speed photogrammetry and optical triangulation. Its near-infrared optical tracking system uses reflective balls as markers and a near-infrared filter attached to lenses to obtain images which only contain the markers. The optical tracker's near-infrared cameras are "blind" to the environment. There is no redundancy when ambient light influences the reflection light from the targets (Greenway, 2000).

\subsection{Design of a 7-D Measurement System}

Considering the measurement requirements for the positional accuracy test method, a 7-D measurement system is being developed by NIST to support this research effort. A visionbased design is selected because 1) Vision-based systems can obtain the positions and orientations information simultaneously; 2) Camera technology is maturing to deliver sub-pixel accuracy. After optical triangulation, the sub-pixel accuracy provides the measurement system with a higher degree of accuracy than what was previously available; and 3) Vision systems are relatively easy to integrate.(Švaco, Šekoranja, Šuligoj, \& Jerbić, 2014).

Instead of using the near-infrared cameras, high speed color cameras are selected. With new, advanced color image stereo technology, target detection can be more accurate by utilizing redundant information from color images. The 7-D measurement system is designed as shown in Figure 4. It consists of two high-speed color cameras, a high performance image processing control box (computer), special targets, and software tools.

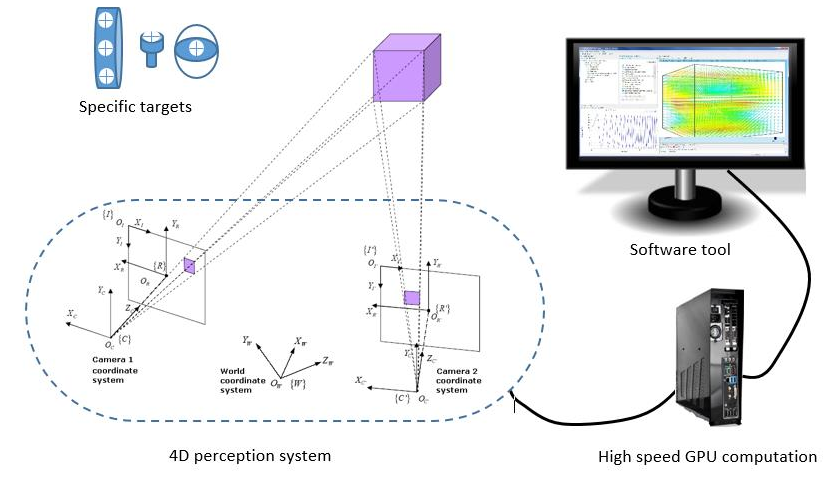

Figure 4. 7-D measurement system

Innovative target design is an important part of this work. Specified targets are designed as adaptors to mount on the robot arm's end-effector with known offsets from the TCP. The purpose of designing innovative target fixtures is to avoid tool changes during measurement that greatly simplified the setup time which requires a brief interruption of the production. By measuring three or more points (that are non-linear, nor in the same plane) on the special target, a coordinate system is established, that contains X, Y, Z, pitch, yaw, and roll information.

The 7-D measurement system is designed with several features which differ from and exceed the performance of traditional stereo technology. These features are the following:

- A time synchronization feature that is designed and embedded within the system.

- A self-calibration feature within the algorithms that utilizes the designed target pattern accuracy (the same target is used for TCP measurement). This feature is to avoid the condition where a camera-based measurement system needs to frequently self-calibrate.

- An advanced color sensor processing feature. Instead of using the near infrared cameras which are 'blind' to the environment, color cameras are used which contain redundant information of the environment conditions for accurate target detection. 
- A fast image processing feature. This enables the complex image processing algorithm to be implemented at a high speed with the graphics processing unit (GPU) programming.

The 7-D measurement system will be mounted on the floor or table to measure the TCP positions. No alignment is needed from the 7-D system to the robot. So the 7-D system can be moved to other stations without the expensive time cost of setup to assess the robot health status using the quick health assessment method. Outputs from the 7-D measurement system are points (time, X, Y, Z, pitch, yaw, and roll) under the fixed instrument coordinate system. The 7-D measurement system captures the fixed loop motion of the robot arm. Analysis is performed to assess the health of the robot system, with an emphasis on the subset of the robot health performance metrics - static accuracy (accuracy of position) and dynamic accuracy (accuracy of trajectory/velocity).

At the conclusion of the test method, the following analyses are completed, including position deviation, orientation deviation, trajectory deviation, and the degradation of these accuracies compared with historical data. The results can be used in the following ways:

1. Yield baseline of the robot system's health condition when a batch of new robot programs is generated. The baseline of positional accuracy health status indicates the health condition when the robot was assigned new tasks, via updated programs. When the robot's health deviates from its baseline condition under the new suite of programs, the prior set of programs needs to be examined and possibly modified (to compensate for any health deviations). Extreme cases may dictate that the system be pulled offline for maintenance.

2. Quick health assessment before a production cycle. The positional accuracy test methods are performed and compared with the baseline to determine both the current health state and ascertain its level of degradation since its baseline test (or last quick health assessment).

3. Serve as an original baseline of a working system's health condition. When a robot has faults/damages that indicate it needs to be replaced, this check is performed to detect if the robot's performance deviates from the baseline. If not, the old program can be run on the replacement robot directly without the need for further intervention (e.g., re-teaching, recalibration, etc.). This check can also be performed in a flexible production environment when new tasks are assigned to different robots.

Assessing the health of the robot system is critical when environmental conditions change or after the work cell has been reconfigured to reduce unexpected downtime, improve productivity, efficiency, and quality, enhance the robot systems' flexibility, and transition maintenance from reactive to preventative to predictive to proactive maintenance.

\section{SUMMARY AND FUTURE WORK}

Manufacturers are constantly expanding their robotic operations within their factory environments to increase their productivity, efficiency, and quality. As such, robot systems are being asked to take on a wide range of tasks, some of which vary over time in reconfigurable environments, where the long-term health prognosis of the overall robot system is largely unknown. As new robot system work cells come online, as their configurations change, or as old components are replaced with new ones, it becomes important to establish the robot system's current and predicted health states under expected future operating conditions. As the state of PHM develops to meet the needs of these emerging robot system work cell configurations, a means of verifying and validating these new PHM capabilities needs to be developed. Numerous research challenges exist in advancing the state of PHM within manufacturing operations. NIST has identified part of the research landscape to address which focuses on developing performance metrics, test methods, reference datasets, and standards/guidelines. These output products will offer manufacturing stakeholders a means to determine the baseline performance of a PHM technology, a means of how that PHM technology performs under varying conditions, and how it compares to other similar implementations. Future efforts are under way to further specify the sensors that will be used to capture data to support robot system health and specify a core group of end-effectors that are representative of those found throughout industry. In concert with further developing the test bed, efforts will also be placed on the next critical step of this research development of the measurement science to determine where faults and failures are originating (given the initial research is focusing on the identification that a fault or failure exists).

\section{NIST DISCLAIMER}

Certain commercial entities, equipment, or materials may be identified in this document in order to illustrate a point or concept. Such identification is not intended to imply recommendation or endorsement by NIST, nor is it intended to imply that the entities, materials, or equipment are necessarily the best available for the purpose.

\section{REFERENCES}

Abdi, H., Nahavandi, S., Frayman, Y., \& Maciejewski, A. A. (2012). Optimal mapping of joint faults into healthy joint velocity space for fault-tolerant redundant manipulators. Robotica, 30, 635-648. doi:10.1017/s0263574711000671

Agheli, M., Qu, L., \& Nestinger, S. S. (2014). SHeRo: Scalable hexapod robot for maintenance, repair, and operations. Robotics and Computer-Integrated Manufacturing, 30(5), 478-488. doi:10.1016/j.rcim.2014.03.008 
Arikan, M. A. S., \& Balkan, T. (2000). Process modeling, simulation, and paint thickness measurement for robotic spray painting. Journal of Robotic Systems, 17(9), 479-494. Retrieved from <Go to ISI > :/WOS:000088830200003

Batzel, T. D., \& Swanson, D. C. (2009). Prognostic health management of aircraft power generators. IEEE Transactions on Aerospace and Electronic Systems, 45(2), 473-483. doi:10.1109/TAES.2009.5089535

Bi, Z. M., \& Lang, S. Y. T. (2007). Automated robotic programming for products with changes. International Journal of Production Research, 45(9), 2105-2118. doi:10.1080/00207540600733634

Bittencourt, A. C. (2012). On Modeling and Diagnosis of Friction and Wear in Industrial Robots. Thesis. Retrieved from liu.divaportal.org/smash/get/diva2:464280/FULLTEXT02. pdf

Buschhaus, A., Blank, A., Ziegler, C., \& Franke, J. (2014). Highly Efficient Control System Enabling Robot Accuracy Improvement. Procedia CIRP, 23, 200205.

doi:http://dx.doi.org/10.1016/j.procir.2014.03.200

Caccavale, F., Marino, A., Pierri, F., \& Ieee. (2010). Sensor Fault Diagnosis for Manipulators Performing Interaction Tasks. Ieee International Symposium on Industrial Electronics (Isie 2010), 2121-2126. Retrieved from $<$ Go to ISI>://WOS:000295007802102

Chen, H., Fuhlbrigge, T., \& Li, X. (2008). Automated industrial robot path planning for spray painting process: a review. Paper presented at the Automation Science and Engineering, 2008. CASE 2008. IEEE International Conference on.

Chen, S. B., \& Lv, N. (2014). Research evolution on intelligentized technologies for arc welding process. Journal of Manufacturing Processes, 16(1), 109122. doi:10.1016/j.jmapro.2013.07.002

Denkena, B., Litwinski, K. M., Brouwer, D., \& Boujnah, H. (2013). Design and analysis of a prototypical sensory Z-slide for machine tools. Production Engineering, 7(1), 9-14. doi:10.1007/s11740-0120419-1

DeVlieg, R. (2010). Expanding the use of robotics in airframe assembly via accurate robot technology. SAE Int. J. Aerospace, 3(1), 198-203.

Edinbarough, I., Balderas, R., \& Bose, S. (2005). A vision and robot based on-line inspection monitoring system for electronic manufacturing. Computers in Industry, $\quad 56(8-9)$, 986-996. doi:http://dx.doi.org/10.1016/j.compind.2005.05.02 $\underline{2}$

ElMaraghy, H. A. (2005). Flexible and reconfigurable manufacturing systems paradigms. International
Journal of Flexible Manufacturing Systems, 17(4), 261-276. doi:10.1007/s10696-006-9028-7

Greenway, B. (2000). Robot accuracy. Industrial Robot, 27(4), 257-265. doi:10.1108/01439910010372136

Holland, S. W., Barajas, L. G., Salman, M., \& Zhang, Y. (2010). PHM for Automotive Manufacturing \& Vehicle Applications. Paper presented at the Prognostics \& Health Management Conference, Portland, Oregon.

Hu, S. J., \& Koren, Y. (1997). Stream-of-variation theory for automotive body assembly. CIRP AnnalsManufacturing Technology, 46(1), 1-6.

IFR, I. F. o. R. (2015). Industrial Robot Statistics. Retrieved from http://www.ifr.org/industrial-robots/statistics/

Jeffries, K. A. (2013). Enhanced Robotic Automated Fiber Placement with Accurate Robot Technology and Modular Fiber Placement Head. SAE International Journal of Aerospace, 6(2), 774-779. doi:10.4271/2013-01-2290

Jin, X., Siegel, D., Weiss, B. A., Gamel, E., Wang, W., Lee, J., \& Ni, J. (2016). The Present Status and Future Growth of Maintenance in US Manufacturing: Results from a Pilot Survey. Manufacturing Review.

Kahan, T., Bukchin, Y., Menassa, R., \& Ben-Gal, I. (2009). Backup strategy for robots' failures in an automotive assembly system. International Journal of Production Economics, 120(2), 315-326. doi:http://dx.doi.org/10.1016/j.ijpe.2007.09.015

Kusuda, Y. (1999). Robotization in the Japanese automotive industry. Industrial Robot, 26(5), 358-360. doi:10.1108/01439919910283786

Lee, J., Wu, F., Zhao, W., Ghaffari, M., Liao, L., \& Siegel, D. (2014). Prognostics and health management design for rotary machinery systems-Reviews, methodology and applications. Mechanical Systems and Signal Processing, 42(1), 314-334.

Liu, G. (2001). Control of robot manipulators with consideration of actuator performance degradation and failures. Paper presented at the Robotics and Automation, 2001. Proceedings 2001 ICRA. IEEE International Conference on.

Malinowski, M., Beling, P., Haimes, Y., LaViers, A., Marvel, J., \& Weiss, B. (2015). System Interdependency Modeling in the Design of Prognostic and Health Management Systems in Smart Manufacturing. Paper presented at the Annual Conference of the Prognostics and Health Management Society 2015, Coronado, CA. http://www.nist.gov/manuscriptpublication-search.cfm?pub_id=918960

Massi, F., Bouscharain, N., Milana, S., Le Jeune, G., Maheo, Y., \& Berthier, Y. (2014). Degradation of high loaded oscillating bearings: Numerical analysis and comparison with experimental observations. Wear, 317(1-2), 141-152. doi:10.1016/j.wear.2014.06.004

Mitsi, S., Bouzakis, K. D., Mansour, G., Sagris, D., \& Maliaris, G. (2004). Off-line programming of an 
industrial robot for manufacturing. The International Journal of Advanced Manufacturing Technology, 26(3), 262-267. doi:10.1007/s00170003-1728-5

Muller, R., Esser, M., \& Vette, M. (2013). Reconfigurable handling systems as an enabler for large components in mass customized production. Journal of Intelligent Manufacturing, 24(5), 977-990. doi:10.1007/s10845-012-0624-y

National Institute of Standards and Technology. (2015, June 2015). Measurement Science Roadmap for Prognostics and Health Management for Smart Manufacturing Systems. Retrieved from http://www.nist.gov/el/isd/upload/MeasurementScience-Roadmapping-Workshop-Final-Report.pdf

Ngan, C.-C., \& Tam, H.-Y. (2004). A non-contact technique for the on-site inspection of molds and dies polishing. Journal of Materials Processing Technology, 155-156, 1184-1188. doi:http://dx.doi.org/10.1016/j.jmatprotec.2004.04. $\underline{263}$

Niku, S. (2011). Introduction to robotics: analysis, systems, applications (2nd ed.): Hoboken, NJ : Wiley, c2011.

Ogbemhe, J., \& Mpofu, K. (2015). Towards achieving a fully intelligent robotic arc welding: a review. Industrial Robot-an International Journal, 42(5), 475-484. doi:10.1108/ir-03-2015-0053

Pan, Z., Polden, J., Larkin, N., Van Duin, S., \& Norrish, J. (2012). Recent progress on programming methods for industrial robots. Robotics and ComputerIntegrated Manufacturing, 28(2), 87-94. doi:http://dx.doi.org/10.1016/j.rcim.2011.08.004

Parhami, B. (1997). Defect, fault, error, ..., or failure? Ieee Transactions on Reliability, 46(4), 450-451. doi:10.1109/tr.1997.693776

Park, C., \& Park, K. (2008). Design and kinematics analysis of dual arm robot manipulator for precision assembly. Paper presented at the Industrial Informatics, 2008. INDIN 2008. 6th IEEE International Conference on.

Shen, T., Wan, F., Cui, W., \& Song, B. (2010). Application of prognostic and health management technology on aircraft fuel system. Paper presented at the 2010 Prognostics and System Health Management Conference, PHM '10, Macau, China.

Shirinzadeh, B. (2000). Repeatability and accuracy - who cares and why? Industrial Robot, 27(4), 250-251. Retrieved from <Go to ISI $>$ ://WOS:000088571800002

Siegel, D., Lee, J., \& Dempsey, P. (2014). Investigation and Evaluation of Condition Indicators, Variable Selection, and Health Indication Methods and Algorithms For Rotorcraft Gear Components. Paper presented at the MFPT 2014 Conference, Virginia Beach, VA.
Siegel, D., Zhao, W., Lapira, E., AbuAli, M., \& Lee, J. (2014). A comparative study on vibration - based condition monitoring algorithms for wind turbine drive trains. Wind Energy, 17(5), 695-714.

Summers, M. (2005). Robot capability test and development of industrial robot positioning system for the aerospace industry. SAE transactions, 114(1), 11081118.

Švaco, M., Šekoranja, B., Šuligoj, F., \& Jerbić, B. (2014). Calibration of an Industrial Robot Using a Stereo Vision System. Procedia Engineering, 69, 459-463. doi:http://dx.doi.org/10.1016/j.proeng.2014.03.012

Tsui, K. L., Chen, N., Zhou, Q., Hai, Y., \& Wang, W. (2015). Prognostics and Health Management: A Review on Data Driven Approaches. Mathematical Problems in Engineering, 2015, 1-17. doi:10.1155/2015/793161

Vijayaraghavan, A., Sobel, W., Fox, A., Dornfeld, D., UC Berkeley, \& Warndorf, P. (2008). Improving machine tool interoperability using standardized interface protocols: MT connect. Proceedings of 2008 International Symposium on Flexible Automation, June.

Wang, R., Liu, L., \& Xu, F. (2008). Research on prognostics technology of robot system. Machine Tool and Hydraulics, 30(11), 15-19. Retrieved from ir.sia.ac.cn/bitstream/173321/.../JXGY200811005.p df

Weiss, B. A., Vogl, G. W., Helu, M., Qiao, G., Pellegrino, J., Justiniano, M., \& Raghunathan, A. (2015). Measurement Science for Prognostics and Health Management for Smart Manufacturing Systems: Key Findings from a Roadmapping Workshop. Paper presented at the Annual Conference of the Prognostics and Health Management Society 2015, Coronado, CA.

Wilson, M. (1999). Vision systems in the automotive industry. Industrial Robot, 26(5), 354-357. doi:10.1108/01439919910283768

Yamada, A., \& Takata, S. (2002). Reliability Improvement of Industrial Robots by Optimizing Operation Plans Based on Deterioration Evaluation. CIRP Annals Manufacturing Technology, 51(1), 319-322. doi:10.1016/s0007-8506(07)61526-1

Young, K., \& Pickin, C. G. (2000). Accuracy assessment of the modern industrial robot. Industrial Robot-an International Journal, 27(6), 427-436. doi:10.1108/01439910010378851

Zhang, X. P., Yan, W. C., Zhu, W., \& Wen, T. (2012). A Design of End Effector for Measuring Robot Orientation Accuracy and Repeatability. In R. Zhu (Ed.), Applied Mechanics and Civil Engineering (Vol. 137, pp. 382-386). 


\section{BIOGRAPHIES}

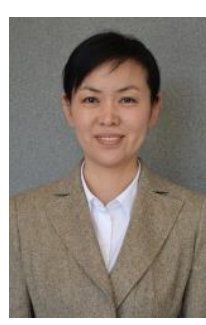

Dr. Guixiu Qiao is a mechanical engineer at the NIST. She is a member of the PHM4SMS project, focusing on PHM for robot systems. She has $12+$ years' experience in high precision industry, expertise in advanced sensors, automation, manufacturing, and information integration; with a strong background in algorithms and optimization. She is a 2011 R\&D 100 award winner for "3D volumetric accuracy error compensation for large machine tool" recognizing the most technologicallysignificant products introduced into the marketplace; 2009 recipient of the Defense Manufacturing Excellence award for the contribution of pioneering efforts in volumetric accuracy for large machine tools; and a 2003 recipient of Boeing's Exceptional Performance award. Dr. Qiao holds her Ph.D. in robotics from the Robot Research Institute, Harbin Institute of Technology, China.

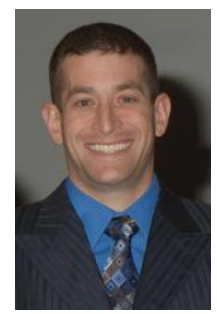

Dr. Brian A. Weiss has a B.S. in Mechanical Engineering (2000), Professional Masters in Engineering (2003), and Ph.D. in Mechanical Engineering (2012) from the University of Maryland, College Park, Maryland, USA. He is currently the Associate Program Manager of the Smart Manufacturing Operations Planning and Control program and the Project Leader of the Prognostics and Health Management for Smart Manufacturing Systems project within the Engineering Laboratory (EL) at NIST. Prior to his leadership roles in the SMOPAC program and the PHM4SMS project, he spent 15 years conducting performance assessments across numerous military and first response technologies including autonomous unmanned ground vehicles; tactical applications operating on Android $^{\mathrm{TM}}$ devices; advanced soldier sensor technologies; urban search and rescue robots; and bomb disposal robots. His efforts have earned him numerous awards including a Department of Commerce Gold Medal (2013), Silver Medal (2011), Bronze Medals (2004 \& 2008), and the Jacob Rabinow Applied Research Award (2006). 